\title{
Contribution of the marketing communication strategy to the hospital performance of healthcare institutions in the city of Lubumbashi
}

\author{
Apport de la stratégie de communication marketing à la performance hospitalière des institutions de soins de santé \\ dans la ville de Lubumbashi \\ Kasongo Ndala John ${ }^{1 *}$, Ditend Yav ${ }^{2}$, Didier Van Caillie ${ }^{3}$, Mundongo Tshamba $^{4}$ and Malonga Kaj ${ }^{4}$ \\ 1,2 Université de Lubumbashi, Faculté des Sciences Economiques et de Gestion, RDC \\ ${ }^{3} \mathrm{HEC}$ Liège, Management School, Liège Université, Belgique \\ ${ }^{4}$ Université de Lubumbashi, Faculté de Médecine et École de Santé Publique, RDC \\ Received 01 March 2019, Accepted 01 May 2019, Available online 02 May 2019, Vol.7 (May/June 2019 issue)
}

\begin{abstract}
The practice of marketing communication has long remained almost non-existent in the health care management sector for essentially ethical reasons, as long as the demand for the health care service was greater than its offer. Healthcare institutions did not feel the need to develop strategies to attract and retain patients. And yet, health organizations are a service provider that requires as much as other areas, the application of management science, including marketing. Changes in the modern social and economic environment require all service providers to behave and market-oriented to adapt to their respective markets, increasingly competitive markets. And one of the axes of this adaptation is their openness to marketing communication strategies... That is why, in this article, we want to reflect on the issues that today constitute the application of marketing communications strategies by health care institutions. This explanatory study was carried out on the contribution of the marketing communication strategy to performance in public and private hospitals in Lubumbashi city. The results of this study indicate that the appropriation of the marketing communication strategy has a positive and significant impact on the hospital performance (24\% of the link rate between the marketing communication strategy and the hospital performance). In addition, the contextual pressure has a positive and significant impact on the success of the marketing communication strategy and the performance of the health care institutions $12 \%$ of the link rate between the environmental context and the implementation of the marketing communication strategy). The study highlights the need for healthcare institutions to implement the communication strategy in order to be successful in a dual goal of promoting health and patient satisfaction in addition to a significant competitive advantage.
\end{abstract}

Keywords: Marketing communication strategy, hospital performance, attractiveness and patient satisfaction

\section{Résumé}

La pratique de la communication marketing est restée longtemps quasi inexistante dans le secteur de gestion des institutions de soins de santé pour des raisons essentiellement d'éthique, aussi bien du fait que pendant longtemps la demande du service de soins de santé était supérieure à son offre. Les établissements de soins de santé ne ressentaient pas la nécessité d'élaborer des stratégies pour attirer et fidéliser la patientèle. Et pourtant, les organisations de santé constituent un secteur prestataire de services qui requiert autant que les autres domaines, l'application des sciences de gestion, notamment le marketing. Les mutations que subit l'environnement socio-économique moderne, imposent à tous les prestataires des services un comportement et un esprit marketing pour pouvoir s'adapter à leurs marchés respectifs, marchés qui du reste sont de plus en plus concurrentiels. Et l'un des axes de cette adaptation est leur ouverture aux stratégies de communication marketing. C'est pourquoi dans le cadre de cet article, nous voulons réfléchir sur les enjeux que constitue aujourd'hui l'application des stratégies de communication marketing par les institutions de soins de santé. Cette étude explicative a été réalisée sur la contribution de la stratégie de communication marketing à la performance dans les hôpitaux publics et privés à Lubumbashi. Les résultats de cette étude indiquent que l'appropriation de la stratégie de communication marketing a un impact positif et significatif sur la performance hospitalières (24\% du taux de liaison entre la stratégie de communication marketing et la performance hospitalière). Par ailleurs, la pression contextuelle impacte positivement et significativement sur la réussite de la stratégie de communication marketing et la performance des établissements de soins de santé (12\% du taux de liaison entre le contexte environnemental et la mise en œuvre de la stratégie de communication marketing). L'étude souligne la nécessité pour les établissements de soins de santé de mettre en place la stratégie de communication afin d'être performants dans un double objectif de promotion de la santé et la satisfaction de la patientèle en plus d'un avantage concurrentiel non négligeable.

Mots clés : Stratégie de communication marketing, performance hospitalière, attractivité et satisfaction patientèle. 


\section{Introduction}

Jusqu'il y a peu, on considérait que les établissements sanitaires n'avaient pas à se faire de souci quant à leurs marchés : il leur suffisait d'attendre leurs patients (puisque ceux-ci recouraient directement à eux soit en situation d'urgence soit sur préavis de leur médecin traitant) et de répondre de manière aussi adéquate que possible aux besoins qui surgissaient spontanément.

Aujourd'hui, un établissement sanitaire ne peut plus ignorer son environnement. II se dégage la nécessité, pour assurer sa survie et son développement, d'attirer et fidéliser les patients, d'informer sur ses prestations ou encore d'améliorer son image de marque comme source de promotion de la santé pour les populations. Il doit donc s'intéresser à ce qui ne relève pas uniquement de la santé et se tourner vers les techniques du marketing et de la communication avec sa patientèle ainsi que l'ensemble des bénéficiaires de ses prestations.

Dans l'économie privée, le marketing comprend l'ensemble des techniques orientées vers la satisfaction des besoins des utilisateurs du service. Dans la pratique, les actions marketing des entreprises sont de ce fait centrées sur un objectif primordial « vendre plus et mieux que le concurrent » (Daria Koutaissoff, 2005).

La réflexion peut-elle être la même dans le domaine de la santé ? Un établissement sanitaire peut-il utiliser les techniques du marketing et de la communication de la même manière qu'une entreprise privée? L'apport et le recours aux techniques marketings sont-ils identiques pour un hôpital public que pour une clinique privée ?

Selon Philip Kotler, Kevin Lane Keller, Bernard Dubois et Delphine Manceau (2006) ; l'activité marketing ne se limite pas à l'élaboration d'un produit et au choix d'un prix et d'un mode de distribution. Une entreprise qui veut aller au-delà d'un courant de vente spontané doit concevoir et transmettre des informations à ses clients actuels et potentiels, à ses détaillants, à ses fournisseurs ainsi qu'aux différentes parties prenantes composant son environnement (médias, administration, opinion publique...). La littérature indique que toute entreprise est agent de communication. Pour la plupart d'entreelles, la question n'est pas de savoir s'il faut communiquer, mais de décider quoi dire, à qui, avec quelle fréquence et quels outils.

On considère la communication comme un véritable dialogue entre l'entreprise et ses clients, qui se déroule avant et pendant la vente, pendant et après la consommation. Compte tenu des possibilités offertes par les technologies de l'information, l'entreprise ne doit plus simplement se demander: " comment contacter mes clients?" mais aussi : "comment permettre à mes clients de me contacter?"

Le point de départ d'une réflexion de communication prend la forme d'un audit de toute interaction reliant l'entreprise à son marché. Pour communiquer

*Corresponding author's ORCID ID: 0000-0001-5575-2435 DOI: https://doi.org/10.14741/ijmcr/v.7.3.3 efficacement, il faut comprendre les différents éléments du processus de communication. Le milieu hospitalier se prête à l'extrapolation des techniques issues du monde industriel dans le but d'améliorer sa performance. C'est ainsi que plusieurs approches sont expérimentées dans le secteur de la santé (Mundongo, 2015).

Un responsable marketing doit commencer par définir l'audience à laquelle il souhaite s'adresser. II peut s'agir d'acheteurs actuels ou potentiels, de revendeurs, ou encore de prescripteurs. La cible de communication se distingue de la cible marketing. Le choix de la cible de communication exerce une profonde influence sur ce qu'il faut dire, comment le dire, où et quand le dire, et à qui il faut le dire. L'entreprise doit analyser les différentes composantes de son image auprès de la cible.

D’après Éric Vernette, Marc Filser et Jean-Luc Giannelloni (2008) ; comme toute action résultant d'une décision de gestion, la communication n'est considérée efficace que si elle atteint les objectifs qui lui sont assignés. Ceux-ci peuvent être objectifs et arbitraires. Par exemple, un spot publicitaire ayant permis d'atteindre une notoriété de $65 \%$ en trois mois pourra ainsi être jugé moins efficace qu'une campagne de parrainage sportif pour laquelle ce résultat ne sera que de $50 \%$ en un an. Tout dépend du marché, notamment de son degré, de I'intensité concurrentielle qui y règne et du bruit réalisé en communication par l'ensemble des acteurs présents, de la situation de l'entreprise sur ce marché (leader, challenger ou suiveur) et des dépenses qui ont été engagées.

Mener une campagne publicitaire consiste à planifier dans le temps la conception et la diffusion de messages destinés aux consommateurs actuels ou potentiels d'un produit ou d'une marque ou plus généralement d'une organisation si l'on rentre dans le champ de la communication institutionnelle. II s'agit d'une activité clairement inscrite dans la stratégie marketing de l'entreprise parce qu'elle implique une totale cohérence avec le positionnement voulu et l'ensemble des composantes du marketing mix.

La publicité est à la fois vecteur de communication et vecteur d'influence, ce qui définit deux niveaux de contrôle spécifiques. Comme vecteur de communication, la publicité doit faciliter le contact avec la cible et permettre la transmission sans défaut de toute la substance du message. Il est donc nécessaire de contrôler a priori la création publicitaire pour garantir cette transmission. Comme vecteur d'influence, la publicité doit produire sur la cible visée un certain nombre d'effets. Ceux-ci vont résulter de la synergie entre la qualité du message et celle du plan médias et doivent être facilement mesurables a posteriori. La communication en général, et la publicité en particulier, mettent un certain temps avant d'être efficaces. Les outils de mesure doivent en tenir compte. Enfin, l'effet d'une communication peut être influencé par un certain nombre de facteurs rivaux tels que la demande, la concurrence, et les autres variables d'action marketing. 
Les mesures d'efficacité publicitaire se différencient selon que l'on cherche à anticiper sur les effets d'une campagne (pré-test) ou que l'on cherche à évaluer les effets obtenus par une campagne diffusée (post-test). Les indicateurs utilisés sont de nature cognitive, affective ou comportementale.

Quant à Jean Jacques Lambin et Jacqueline Demorloose (2008) ; la réunion des conditions matérielles de l'échange ne suffit pas à assurer un ajustement précis entre l'offre et la demande. Pour que la rencontre entre les offreurs et les demandeurs puisse s'effectuer, des flux de communication doivent être mis en place entre les différents partenaires dans le processus d'échange, notamment à l'initiative de l'entreprise, en vue de faire connaître au marché le positionnement revendiqué par la marque ou l'entreprise.

Les rôles des vendeurs connaissent des changements importants et la négociation commerciale, ou la vente relationnelle, tend de plus en plus à remplacer les techniques de ventes traditionnelles.
Cette évolution donne aux vendeurs des fonctions importantes dans le domaine du marketing stratégique. Les dépenses consacrées à la promotion des ventes prennent une part croissante du budget total de communication, notamment en raison du développement des techniques du marketing direct. Il existe une grande variété de techniques promotionnelles dont les effets sont complexes, certains risquant même de dévaloriser. Les relations publiques et la communication hors-médias constituent une forme de communication douce qui gagne en popularité dans la mesure où on observe une baisse de l'efficacité de la publicité-produit. Lorsque les entreprises font appel à la publicité média, elles adoptent une stratégie d'aspiration en s'adressant directement aux clients finals.

Partant d'un survol de la littérature, une grille de synthèse des principales thématiques abordées par les auteurs et des tendances marketing dans le secteur de santé a été élaborée:

Tableau 1 : Grille de lecture des tendances marketing dans le secteur de la santé

\begin{tabular}{|c|c|c|c|}
\hline 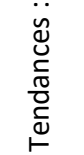 & $\begin{array}{c}\text { L'application des stratégies marketing de } \\
\text { service pour attendre la satisfaction des } \\
\text { patients dans les institutions des soins } \\
\text { de santé }\end{array}$ & $\begin{array}{l}\text { Le marketing social et la } \\
\text { communication dans le domaine de la } \\
\text { santé }\end{array}$ & $\begin{array}{l}\text { Stratégies de réorganisation } \\
\text { des organisations } \\
\text { hospitalières : tendance de la } \\
\text { réforme par le marketing }\end{array}$ \\
\hline 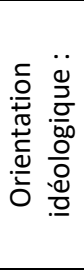 & $\begin{array}{l}\text { Ces auteurs préconisent le recours aux } \\
\text { techniques du marketing de service au } \\
\text { sein des institutions hospitalières } \\
\text { comme vecteur pour atteindre la } \\
\text { satisfaction des patients et pérenniser la } \\
\text { structure. }\end{array}$ & $\begin{array}{c}\text { Cette tendance préconise l'intégration } \\
\text { du marketing dans le domaine social } \\
\text { par l'usage des stratégies de } \\
\text { communication afin de bénéficier de la } \\
\text { notoriété et de l'image nécessaire pour } \\
\text { l'acquisition des avantages } \\
\text { concurrentiels }\end{array}$ & $\begin{array}{l}\text { Pour ce courant, le recours au } \\
\text { marketing s'effectue dans le } \\
\text { cadre de la réforme } \\
\text { hospitalière afin de réduire } \\
\text { les coûts et atteindre une } \\
\text { certaine performance dans } \\
\text { ces institutions. }\end{array}$ \\
\hline 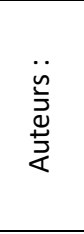 & $\begin{array}{c}\text { Zakia Kessas(2011) } \\
\text { Gendouzzi Brahim (2015) } \\
\text { Anouk Grevin(2011) } \\
\text { Nicolas Petit (2013) } \\
\text { Michel Baellen (1996) } \\
\text { Géraldine Prunet (1999) }\end{array}$ & $\begin{array}{l}\text { Jacques De Guise(1995) } \\
\text { Durigitto Fabio(2010) } \\
\text { Stéphane Aubert(2008) } \\
\text { Véronique Serry (2015) } \\
\text { Bernard Dubois(1987) }\end{array}$ & $\begin{array}{l}\text { Frédérique Quidi (2015) } \\
\text { Sophie Piault (2006) }\end{array}$ \\
\hline
\end{tabular}

\section{Méthodologie}

Cette étude s'inscrit dans le paradigme interprétativiste, c'est-à-dire, nous cherchons à comprendre la signification que les acteurs des institutions hospitalières dans les zones de santé de Lubumbashi donnent à la communication marketing, leurs motivations et la performance de leurs organisations. Notre but n'a pas été de découvrir des lois qui s'imposent aux acteurs, mais de chercher à décrire comment les acteurs construisent le sens qu'ils donnent à la communication marketing et la performance organisationnelle.

Nous pensons ainsi que la méthode proposée par Huberman et Miles (1991), même si ces auteurs se qualifient des "positivistes modérés " (Koenig, 1993), s'applique très bien dans un paradigme interprétativiste, car la méthode qu'ils proposent permet de rester le plus fidèle possible aux interprétations que possèdent les acteurs de leur situation. Notre population d'étude a été constituée par les managers des institutions hospitalières privées et publiques situées dans la ville de Lubumbashi en tant qu'acteurs de la stratégie de communication marketing d'une part, mais aussi des flux des patients fréquentant ces centres en tant que cible de la communication marketing, d'autre part.

Compte tenu de la spécificité sur l'objet de l'étude, concernant la compréhension de la relation entre l'appropriation des stratégies de communication marketing et la performance des établissements de soins de santé dans la ville de Lubumbashi, nous nous sommes référés à la base des données que nous a fourni la direction provinciale de la santé en rapport avec le nombre des établissements de soins de santé au cours l'année 2017 qui se chiffrait à 317 (population mère). 
Nous avons ciblé en premier temps les responsables des directions et leurs adjoints et ensuite les différents agents au sein des ce même direction. Le choix de la taille de l'échantillon a été dicté par les saturations des variables contenues dans les hypothèses de cette recherche. Nous sommes entretenus avec 395 répondants qui ont constitués la base des données qui a été traité par l'analyse du contenu à l'aide de logiciel Tropes. Le modèle théorique retenu dans l'étude est le suivant :

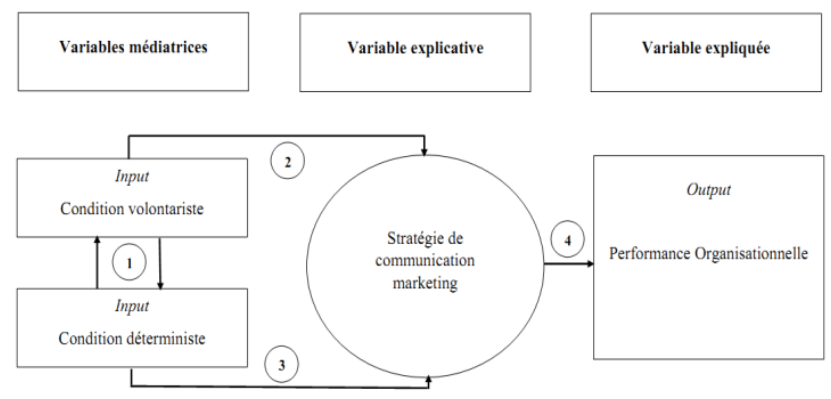

Fig. 1 : Modèle théorique de l'étude
Le modèle met en relation la stratégie de communication marketing à la performance hospitalière des institutions de soins de santé dans la ville de Lubumbashi passant par deux conditions nécessaires et suffisante, à savoir : La condition volontariste (qui est fonction de la structure organisationnelle de l'établissement de soins de santé) et la condition déterministe (qui est fonction de l'environnement dans lequel l'établissement de soins de santé évolue).

\section{Résultats}

Après avoir soumis les données secondaires des entretiens au logiciel Tropes sous la forme d'un fichier word qui affiche les résultats sommaires en termes des propositions remarquables par rapport aux scénarios des entretiens à l'aide de l'analyse cognitivo-discursive, dix univers de référence ont été retenus en fonction de leurs occurrences, à savoir :

Tableau 1 : Résultat numérique des entretiens qualitatifs

\begin{tabular}{|c|c|c|}
\hline Univers des références & Occurrence en V.A+ & Taux de liaison=Occurrence/Tot \\
\hline Communication & 20 & $24 \%$ \\
\hline Rivalité (concurrence) & 10 & $12 \%$ \\
\hline $\begin{array}{c}\text { Avantages concurrentiels } \\
\text { /compétitivité }\end{array}$ & 8 & $9,25 \%$ \\
\hline Stratégie & 8 & $9,25 \%$ \\
\hline Performance organisationnelle & 8 & $9,25 \%$ \\
\hline Résultat & 8 & $9,25 \%$ \\
\hline Qualité & 6 & $7 \%$ \\
\hline Prix & 6 & $7 \%$ \\
\hline Infrastructure & 6 & $7 \%$ \\
\hline Procédures & 5 & $6 \%$ \\
\hline Total & 85 & $100 \%$ \\
\hline
\end{tabular}

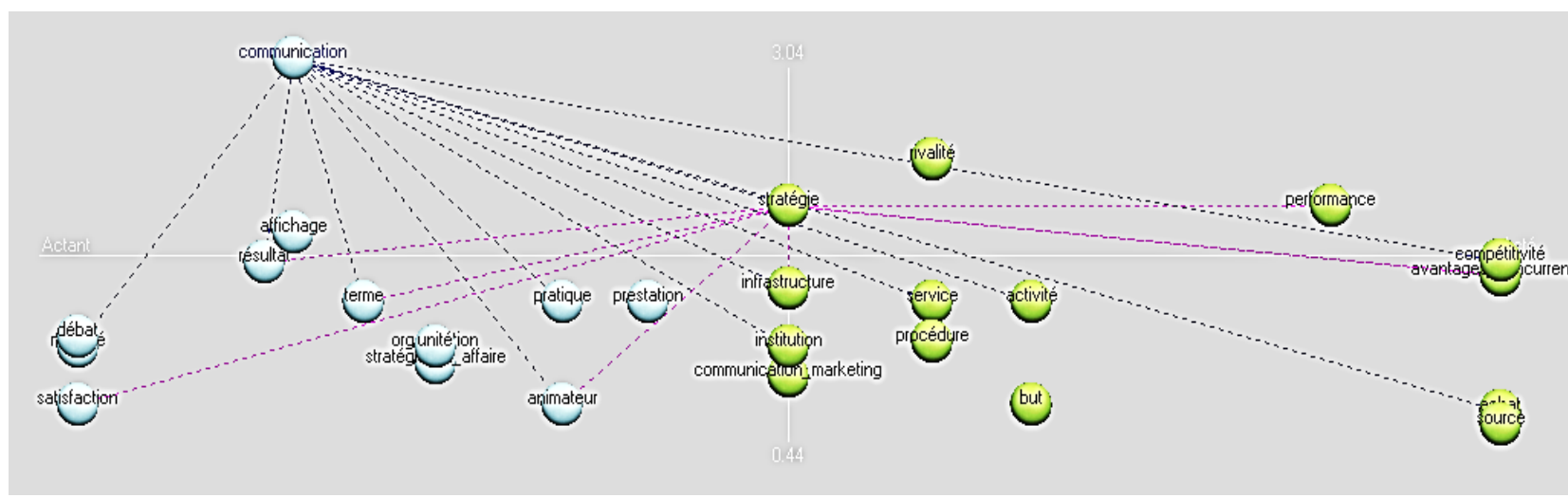

Source : analyse des données par le logiciel Tropes

Fig. 2 : Liens entre la stratégie de communication marketing et la performance hospitalière

Le tableau ci-haut indique les univers des références contenant les indicateurs qui ont été retenus dans le modèle de l'étude qui correspond à la variable correspondante ressortie dans les entretiens divisé par le nombre total des variables. Ce qui a permis de dégager le taux de liaison correspondant par rapport à chacune des références indiquées.

La communication marketing affiche un taux de liaison majeure (24\%) sur l'ensemble des références retenues, vient ensuite la concurrence qui se positionne en seconde 
place avec $12 \%$ et enfin le huit autres références qui se répartissent $74 \%$ du taux de liaison avec la performance hospitalière des établissements de soins de santé dans la ville de Lubumbashi.

Partant des univers de références obtenus dans nos résultats graphiques, les graphes ci-après ont été générés par Tropes expliquant les relations entre les variables et les indicateurs :

Partant du graphe des acteurs, on constate la concentration des relations de la majorité des variables sur la communication marketing (variable explicative) :

- Trois variables clés sont étroitement corrélée à savoir la stratégie de communication marketing, la rivalité et la performance hospitalière (variables proches de celle déterminante) avant de déboucher sur la compétitivité et les avantages concurrentiels.

- Le logiciel a placé la variable principale en haut à gauche comme étant sujet (actant) ayant une forte concentration des relations (la communication) considérée comme étant l'activité principale sur laquelle tournent les stratégies des institutions de soins de santé dans la ville de Lubumbashi. En bas du cadrant à gauche nous retrouvons la satisfaction de la patientèle considérée comme l'objectif de la mise en œuvre de cette stratégie afin d'atteindre le résultat.

Partant de l'analyse qualitative, les résultats obtenus peuvent se résumer comme suit:

1. Il existe une relation positive entre la stratégie de communication markéting et la performance des institutions de soins de santé dans la ville de Lubumbashi. Cette relation peut s'expliquer sur deux volets :

- Les structures qui pratiquent la communication bénéficient de la compétitivité par rapport à celles qui ne la pratiquent pas, et cela s'affiche en termes de la performance hospitalière et des avantages concurrentiels,

- Cette stratégie de communication marketing est influencée par le contexte concurrentiel dans lequel évoluent ces établissements de soins de santé.

2. La mise en œuvre de la stratégie de communication marketing permet à ces dernières de mettre en phase leurs infrastructures, le processus et la qualité des services dans l'intérêt de la satisfaction de la patientèle ;

3. Cette communication marketing permet aux institutions de soins de santé de s'aligner par rapport à leur environnement interne, externe et interactif ;

4. Le bouche à oreille (marketing direct) apparait comme un vecteur prioritaire dans la mise en œuvre de la communication marketing au sein des institutions de soins de santé dans la ville de Lubumbashi.
5. L'intensité de la communication marketing au sein de ces structures varie d'une institution à l'autre en fonction des variables telles que :

- La taille de l'institution et l'infrastructure hospitalière disponible ;

- La rivalité concurrentielle dans la zone ;

- Le secteur auquel appartient la structure (publique ou privée) ;

- Le niveau de vie de la patientèle se trouvant dans les différentes zones de chalandise de ces institutions.

\section{Discussion}

Considérant les littératures documentées sur le sujet et en les comparants aux résultats auxquels cette étude a abouti, nous constatons une convergence.

Certains auteurs conditionnent la performance hospitalière par la mise en œuvre par l'établissement de soins de santé d'une stratégie de communication accès sur la qualité de soins (DE GUISE, 1995 ; KESSAS, 2011 ; QUIDI, 2015).

D'autres complètent l'approche de cette étude d'une manière globale en mettant en exergue la relation positive entre la performance hospitalière dans les établissements de soins de santé et les pratiques du marketing au sein de ces dernières (PIAULT, 2006 ; FABIO, 2010 Sery, 2015 ; BRAHIM, 2015).

Les résultats de ces études antérieures rencontrent les préoccupations de la présente étude qui complète par une approche qualitative les observations et établit un liaison entre la stratégie de communication marketing et la performance hospitalière des institutions de soins de santé dans la ville de Lubumbashi.

Les stratégies de communication marketing rencontrent les préoccupations de plan stratégique recommandé dans le secteur de la santé, qui prône la combinaison de $4 \mathrm{P}$ (La qualité du Produit, la Place de production des soins qui assurent l'accessibilité géographique, la Promotion des prestations de soins à l'égard des bénéficiaires et le Prix qui prend en compte l'accessibilité financière de la population) stratégique pour le positionnement de l'établissement de santé par rapport à ces concurrents.

Cette stratégie de marketing mix permet à l'établissement de soins de santé de rencontrer, d'une part, les orientations de l'OMS, en ce qui concerne l'accessibilité géographique et financière, et d'autre part, le devoir pour les établissements de soins de santé d'assurer la production des soins de qualité dans l'optique des approches de l'amélioration continue par l'assurance qualité. A ce propos, aucun établissement des soins de santé en quête d'une performance ne peut négliger la mise en place des stratégies de communication avec ses parties prenantes afin de saisir les attentes et améliorer sa notoriété et la qualité de ses prestations, ce qui lui donne un avantage concurrentiel important par rapport à ses concurrents et vis-à-vis de sa clientèle. 
En ce qui concerne la place de la clientèle dans la stratégie de communication marketing, les expériences à travers les entreprises de production des biens ont mis en relief le rôle combien important que joue cette approche et son impact sur les consommateurs des produits.

Les professionnels du management travaillant dans le domaine hospitalier font face à plusieurs défis, notamment ceux concernant les failles du néo management organisationnel: a) pathologie de la communication, b) injonctions paradoxales, c) productivité et rythme de travail, et d) adhésion affective. Les nouveaux aspects déterminant le contexte hospitalier (rôle actif du patient, développement technologique, etc.) rendent plus compliquée l'application des théories traditionnelles du management. Dans ce contexte, la promotion de la dimension communicationnelle du management hospitalier pourrait permettre à ces institutions de résoudre quelques-unes de ces failles, de mettre en valeur l'individu -soit, l'employé hospitalier- et d'améliorer ainsi la qualité du service médical offert au patient (Pablo Medina et Ghizlaine Lahmadi, 2019). Ce nouvel axe stratégique permettant alors à ces organisations de mieux satisfaire leurs employés comme leurs patients. La dimension communicationnelle du management hospitalier aide l'organisation à mettre l'individu au centre des procès de travail qui ont lieu à I'hôpital, ce qui semble être en cohérence avec les nouvelles exigences des employés et des patients (DE GUISE, 1995 ; PARSONS, 2001).

Le comportement du consommateur des services dans tous les secteurs, tant commercial que de production des services se caractérise par l'évaluation de la qualité des services offerts par les prestataires. Par conséquent, tous les mécanismes de contact et d'échange avec les consommateurs des prestations des établissements de production des services se révèlent l'une des options stratégiques conditionnant non seulement le positionnement de l'établissement mais aussi lui donnent une plus-value sur la consommation de ses prestations avec comme conséquence économique une amélioration des rentrées financières générées par l'achat des services (Adrien Malandj et al., 2019). Les stratégies d'achat stratégique mises en place dans le secteur de la santé n'ont pas donné des résultats satisfaisants par manque d'un travail réel de promotion de la santé auprès des bénéficiaires, $c^{\prime}$ est-à-dire la clientèle.

Le PBF (Performance based financing) donne des résultats très mitigés à cause $d u$ manque d'accompagnement par les stratégies de communication marketing et les approches d'amélioration qualité par la qualité des prestations hospitalières (Fox S et al., 2013 ; Saidou, 2019)

\section{Limites et Perspectives}

Les limites de cette étude se présentent comme suit:

- Au niveau de notre champ empirique qui est la ville de Lubumbashi, regorgeant des caractéristiques d'une ville urbanisée disposant des structures des soins assez développées par rapport aux zones rurales limitent la généralisation des résultats. Les études sur le même sujet orientées dans des régions rurales pourront compléter nos analyses sur cette thématique aussi importante pour la survie des établissements de soins de santé, qui ont la mission de la promotion de la santé en plus des aspects de médecine curative.

- Sur le plan théorique, la performance hospitalière étant un concept multidimensionnel, les variables retenues dans cette étude sont exhaustives et peuvent varier selon le contexte pouvant ouvrir d'autres questionnements sur le problème.

\section{Conclusion}

Le marketing prépare les dirigeants d'établissements de soins de santé à mieux connaître les besoins et la demande du public, à mieux évaluer l'offre de la concurrence et à mieux anticiper les évolutions du marché.

En d'autres termes, l'objectif du marketing est double ; d'une part, il vise à adapter les services offerts aux besoins des usagers tout en tenant compte des contraintes du marché, et d'autre part, à évaluer en permanence le degré de satisfaction des usagers ainsi que l'image et la notoriété de l'établissement.

Dans l'environnement économique actuel, une institution, publique ou privée, ne peut plus vivre sans se faire connaître, sans faire savoir ce qu'elle est et ce qu'elle désire devenir. Toute institution de santé doit se soucier de générer autour d'elle une aura favorable, de faire connaître son activité, ses résultats, ses espoirs, les recherches qu'elle a entreprise et les résultats qu'elle a obtenus. Une telle attitude de marketing ne visera pas en premier lieu à vendre, mais à communiquer, à influencer favorablement et à améliorer l'image et la notoriété.

Aujourd'hui, on assiste à la prise de conscience de la part des dirigeants des institutions de soins de santé du rôle primordial de la communication dans le secteur de santé afin de renforcer les mécanismes de promotion de la santé, englobant la publicité et l'information. La communication est en effet destinée à devenir de manière généralisée un service, une fonction à part entière dans les établissements de soins de santé. Son existence prouve que l'information est l'élément essentiel de la cohésion de l'ensemble des acteurs de l'établissement et permet la dynamique des objectifs et l'amélioration de la qualité et de la performance hospitalière. Elle est donc indispensable et on attendra de plus en plus de cette activité de véritables résultats comme d'une des fonctions de production au sein des hôpitaux publics et privés dans la ville de Lubumbashi. L'étude indique la nécessité pour les établissements de soins de santé de mettre en place la stratégie de communication afin d'être performants dans un double objectif de promotion de la santé et la satisfaction de la 
patientèle en plus d'un avantage concurrentiel non négligeable.

\section{Conflit d'intérêt}

Les auteurs ne déclarent aucun conflit d'intérêt.

\section{Références Bibliographies}

[1]. ADRIEN MALANDJ KES, MULUBWA KYALUBILE, MUJINGA KASHALA., Patient satisfaction, one of the levers for the health services performance: Contribution to scientific evidence, Lubumbashi, Democratic Republic of Congo, Int. J. of Multidisciplinary and Current research, 2019, Vol 6..

[2]. BOINET S., " un outil de pilotage pour l'établissement de santé ॥, Gestion hospitalière, $\mathrm{n}^{\circ} 547$,pp370-373,2015.

[3]. BARDIER J., "L'hôpital fait peau neuve pour mieux séduire ": Architecture et prise en charge des patients. Hospitals.be, $n^{\circ} 1,2009$.

[4]. BRUNNELLE Y., "les hôpitaux magnétiques : un hôpital où i fait bon se faire soigner "Pratiques et organisation des soins, volume $40, n^{\circ} 1, p p .39-48$.

[5]. CAILLE R., CRIER D., " stratégie du groupe public: Pour un marketing hospitalier ", revue hospitalière de France, $n^{\circ} 525, \mathrm{pp} 18-21,2008$.

[6]. CHANTEUR M., "un nouveau démarche", revue hospitalière de France, $n^{\circ} 525$,pp18-21,2008.

[7]. CHANTEUR M., DUBOIS B., LOUPEE A., ROODENBEKE E., « $i$ était une fois...le GRAHM », revue hospitalière de France, $\mathrm{n}^{\circ} 525, \mathrm{pp} 27-29,2008$.

[8]. CRIE D., " pourquoi et comment la marketing à l'hôpital ? ", Gestion hospitalières, $n^{\circ} 547$, pp 361-364,2015.

[9]. CRIE D., SALERNO D., VINCENT A., "vers un marketing hospitalier ? »Problèmes économiques, $n^{\circ} 311, \mathrm{pp}$ $58,2015$.

[10]. CORRINE J., "Communication externe aux HUG, un outil de démocratie ", in Competence, n³,p.25-27,2003.

[11]. DUBOIS B., "L'hôpital à l'heure du marketing ou la religion à l'usager ", solidarité santé, $n^{\circ} 5$, page 49-60,1988.

[12].DURFOUR P., 2012," Construction d'un score agrégé d'attractivité ", Gestions hospitalières, $n^{\circ} 518$,pp 397406,2012.

[13].FERRARD H., SUDRE M. "Service public et marketing hospitalier le mariage peut-il être consommé ?" Revue marketing $n^{\circ} 522$, Novembre-Décembre, 2008.
[14]. FOX, S., WITTER, S., WYLDE, E., MAFUTA, E., et LIEVENS, T. Paying health workers for performance in a fragmented, fragile state: reflections from Katanga Province, Democratic Republic of Congo. Health Policy and Planning, (2013). 29(1), 96-105.

[15].DE GUISE J., "Marketing social et stratégies de communication dans le domaine de la santé ", Recherches en Communication, 1995, vol. 4, supplément.

[16]. GAILLARD M. "Vous avez dit marketing ? Comment créer de la valeur dans les établissements de santé ". Revue du cadre de santé, n²4, Mai-Juin, 2006.

[17]. GALLOPEL K., "Le marketing hospitalier. Vous avez dit marketing ?oui, marketing ", n547,2015

[18]. HEIM R. "l'attractivité, un enjeu marketing », Gestions hospitalières, $n^{\circ} 547$, pp 377-379,2015.

[19]. MERDINGER C., " Démarche marketing, les enjeux à I'hôpital ", Gestions hospitalières, n519, pp 495-498,2012.

[20]. MUNDONGO T., «La conception et l'implémentation d'une stratégie organisationnelle performante aux Cliniques Universitaires de Lubumbashi en RDC », Thèse de doctorat, Gestion de la performance des établissements de santé, 2015.

[21]. PABLO MEDINA et GHIZLAINE LAHMADI, " La dimension communicationnelle du management hospitalier ", Communication et organisation, 2012, consulté le 19 avri 2019.

[22]. PARSONS P., the healthcare manager's guide to strategic communication, Chicago, Health Administration Press, 2001.

[23].PHILLIPE S., Destination santé; un cas particulier de tourisme sur le littoral lémanique Vaudois: les étrangers soignés dans les hôpitaux publics et les cliniques privées, Université de Lausanne, Institut de Géographie, session octobre 1988.

[24].THIBAULT M., "le marketing hospitalier », objectifs soins, $n^{\circ} 36, p p 31-36,1995$.

[25]. ROBILLARD L., " Le marketing hospitalier un oxymore qui prend de sens ". Revue hospitalière de France, Juillet-Aout, 2007.

[26].SAIDOU H., Recours thérapeutiques en cas de paludisme: impact d'une réforme de l'offre de soins de santé au Cameroun. STATECO, 2018.

[27]. TARDIF L., LOUAZEL M., "Projet d'établissement et marketing hospitalier ", Gestions hospitalières, $n^{\circ} 547, \mathrm{pp}$ 365-368,2015. 